\title{
Paradojas del comportamiento proambiental de los estudiantes universitarios en diferentes disciplinas académicas ${ }^{1}$
}

\section{Paradoxes of the environmental behavior of university students in different academic disciplines}

\author{
Marithza Sandoval-Escobar², Pablo Páramo³ ${ }^{2}$ Johnny Orejuela $^{4}$, Iván González \\ Gallo $^{5}$, Omar Fernando Cortés ${ }^{6}$, Ketty Herrera Mendoza ${ }^{7}$, Carolina Garzón ${ }^{8}$ \\ y Cesar Erazo ${ }^{9}$
}

${ }^{2}$ Doctora, Docente Investigadora, Fundación Universitaria Konrad Lorenz. Bogotá, Colombia. E-mail: marithza.sandoval@konradlorenz.edu.co.

${ }^{3} \mathrm{PhD}$. Docente Investigador, Universidad Pedagógica Nacional de Colombia. Bogotá, Colombia. E-mail: pdeparamo@gmail.com

${ }^{4}$ Doctor, Docente Investigador, Universidad EAFIT

${ }^{5} \mathrm{Mg}$, Docente Investigador, Universidad Autónoma de Bucaramanga, Colombia

${ }^{6}$ Docente Investigador, Universidad Sergio Arboleda, Colombia

${ }^{7}$ Docente Investigador, Universidad de la CostaBarranquilla, Colombia

${ }^{8}$ Docente Investigador, Universidad Santo Tomás, Bogotá, Colombia

${ }^{9}$ Director de Programa, Politécnico Grancolombiano, Medellín, Colombia

${ }^{1}$ El presente artículo ha sido derivado del proyecto de investigación titulado Análisis del Discurso Ambiental Intersectorial en la Educación Superior de Colombia, desarrollado en el Doctorado en Educación de la Universidad Pedagógica Nacional.

\section{Resumen}

El objetivo del presente estudio fue establecer las características del comportamiento proambiental de los estudiantes universitarios colombianos, esto es, identificar las actitudes, creencias, valoraciones, prácticas y conocimientos ambientales de estudiantes colombianos de diferentes carreras universitarias. Para ello se realizó un estudio descriptivo-correlacional de corte transversal con una muestra de 1503 estudiantes de diferentes carreras universitarias en cinco ciudades colombianas, con una probabilidad de inci- 
dencia de .5, $95 \%$ de confianza y un error del $2.5 \%$. Los participantes fueron seleccionados aleatoriamente al interior de cada universidad y seles aplicaron el Inventario de Comportamiento Proambiental (ICP) y el Test de Conocimiento Ambiental (TCA), este último desarrollado específicamente para propósitos de la presente investigación. Se encontró que los estudiantes encuestados poseen actitudes y creencias ambientales positivas en la mayoría de las dimensiones. El nivel de conocimiento ambiental de los estudiantes en las cinco ciudades seleccionadas es bajo. Si bien no se observaron diferencias significativas entre las carreras respecto a las creencias, valoraciones, acciones y conocimientos ambientales, paradójicamente los estudiantes de ciencias ambientales ocuparon lugares inferiores a los que estudian ciencias económicas y administrativas. Se puede concluir que existen diferencias según la ciudad en las creencias, valoración, conocimiento y acciones ambientales de los estudiantes encuestados. El reporte de acciones ambientales presenta un perfil controversial pues al mismo tiempo las personas realizan comportamientos proambientales y antiambientales. Las instituciones universitarias y el gobierno tienen el desafío de lograr que la educación ambiental muestre los resultados esperados para el cumplimiento de las metas ambientales del país y del mundo.

Palabras clave: Comportamiento proambiental; Conocimiento ambiental; Actitudes ambientales; Comportamiento sustentable; Educación superior.

\section{Abstract}

The objective of the present study was to establish the characteristics of the pro environmental behavior of Colombian university students; that is, to identify the attitudes, beliefs, valuations, practices and environmental knowledge of Colombian students from different university careers. For this purpose, a cross-sectional descriptive-correla- tional study was carried out with a sample of 1503 students from different university careers in five Colombian cities, with an incidence probability of .5, 95\% confidence and an error of $2.5 \%$. The participants were randomly selected within each university and the questionnaires applied were the Pro environmental Behaviour Inventory (ICP) and the Environmental Knowledge Test (TCA); the latter was developed specifically for the purposes of this research. The students surveyed were found to have positive environmental attitudes and beliefs in most dimensions. The level of environmental awareness of students in the five selected cities is low. Although no significant differences were observed between the careers with respect to beliefs, valuations, actions and environmental knowledge, paradoxically the students of environmental sciences occupied inferior places to those who study economic and administrative sciences. Thus, in the analysis of specific items it was evidenced that the students of environmental sciences develop diverse anti environmental actions, in a similar way to the behavior patterns of the students of the other researched careers.

With respect to the region, the results show that it is possible to conclude that there are differences according to the city with respect to the beliefs, valuation, knowledge and environmental actions of the students surveyed. Among the findings it is worth highlighting the results of Bucaramanga in terms of the report of environmental actions and the highest average in the TCA observed in that city. Similarly, Cali stands out along with Bucaramanga in its results, while Medellín obtained the lowest scores in environmental knowledge.

Finally, in the report on environmental actions in university students, contradictory results are observed, given that it presents a controversial profile because people perform pro-environmental and anti-environmental behaviors at the same time, with positive environmental attitudes and beliefs in accordance with these attitudes. Regarding the knowledge 
about the environment, in the descriptive results the average of the test was 12 points, which means that the students failed the test. The multivariate analysis showed that the theoretical model on which the construction of the ICP was based and the instruments from which items were selected for its conformation, apparently has great predictive power with respect to the verbal report of environmental actions; however, the TCA, a test constructed specifically for this research, would covariate with almost all the factors of the ICP but does not predict causally the actions reported by the students.

In general, this study shows that university institutions and the Colombian government have the challenge of ensuring that environmental education achieves the expected results for the fulfillment of both the country and the world's environmental goals. The results also demonstrates the need to articulate policies, actions and regulations to improve the quality of environmental education in universities, as well as to implement citizen behavior programs that promote responsible consumption, given that knowledge does not correlate with effective actions in terms of public services, green brands and solid urban waste management, among other areas.

Keywords: Pro-environmental behavior; Environmental knowledge; Environmental attitude; Sustainable behavior; Higher education.

\section{Introducción}

En Colombia, la política nacional ambiental determina que las instituciones universitarias tienen como función lograr la pertinencia de los procesos educativos para aportar a la comprensión y solución de la problemática ambiental, propiciando la participación social y contribuyendo a la solución de los problemas socioambientales. Con ese fin se han propuesto los PRAU (Proyectos Ambientales Universitarios) los cuales se desarrollan en contextos ambientales, sociales, económicos y culturales particulares. Dentro de la política nacional ambiental se asume que los PRAU deben ser fundamentados en conceptos sistémicos del ambiente e incorporados como parte del Proyecto Educativo Institucional (PEI), involucrando a la comunidad y a los egresados dentro de los programas. Los estudiantes universitarios, como cualquier otro ciudadano, están obligados a mostrar un patrón de comportamiento acorde con los objetivos ambientales del país y las instituciones educativas, de acuerdo con las políticas ambientales de Colombia, deben destinar esfuerzos para lograr estos objetivos, a partir de la investigación, la formación de los estudiantes y la educación ambiental de la comunidad universitaria. A pesar de que existe una política ambiental y los mecanismos para que se implemente en las universidades, muchos indicadores de comportamiento proambiental en las ciudades colombianas hacen suponer que algo está fallando.

Aun cuando se ha observado un crecimiento del $56 \%$ en el número de carreras ambientales en el país en los últimos diez años, y una marcada reducción de los pregrados en el área de explotación de recursos agrícolas y forestales, los resultados ambientales del país respecto del comportamiento de los ciudadanos no muestran un cambio significativo (Cortés-Peña, 2011a, 2016a; Torres-Hernández, Barreto y Rincón, 2015). Esto puede relacionarse con los problemas en la implementacón de la educación ambiental al interior de las universidades. El diagnóstico realizado por Román (2014) sobre la inclusión de la dimensión ambiental en algunas universidades de Bogotá (Colombia), mostró que desde la alta dirección existe un fuerte compromiso con el ambiente, un crecimiento de electivas en ambiente y el surgimiento de grupos de investigación con proyectos ambientales. Solo una de las universidades analizadas poseía indicadores de seguimiento para la inclusión de la dimensión ambiental en el currículo a partir de indicadores de compor- 
tamiento proambiental de la comunidad universitaria. Ninguna de ellas reportó la existencia de PRAU en las estrategias de educación ambiental de sus comunidades y fue evidente que pocas implementaban procesos de manejo de residuos sólidos urbanos, entre otras estrategias de gestión ambiental interna.

Otro aspecto importante a tener en cuenta se relaciona con las dimensiones del comportamiento proambiental y aquellas que se relacionan directamente con los indicadores de la educación ambiental. Los modelos tradicionales se han centrado en las actitudes y la intención conductual, las cuales han recibido múltiples cuestionamientos en virtud de su bajo poder predictivo respecto del comportamiento efectivo (Amérigo, 2006; Berenguer y Corraliza, 2000).

Los cuestionamientos anteriores han incidido en la adopción de otro tipo de modelos para explicar el comportamiento proambiental, los cuales relacionan las creencias de las personas y su contexto social con las acciones efectivas; tal es el caso del modelo TAR (Teoría de la Acción Razonada) (Ajzen y Fishbein, 2005; Fishbein y Ajzen, 2011) y la Teoría de la Conducta Planeada de Ajzen (1991, 2002). La TAR asume que las creencias, actitudes, intenciones y comportamientos de los individuos determinan el proceso de decisión de las personas en distintos ámbitos, asumiendo que no siempre existe una correlación entre actitud y comportamiento efectivo. La intención depende también de las influencias sociales que experimente el individuo en su entorno y cómo él perciba las presiones que ejerce el medio social para que ejecute un comportamiento (norma subjetiva). Tanto las creencias como las conductas influyen en la predicción del comportamiento efectivo, pero no forman parte de la actitud. Por su parte, la TCP involucra un factor adicional para mejorar la predicción de la conducta efectiva, como es el de control percibido de la conducta, el cual tiene un efecto directo sobre la intención conductual. En cualquier caso, es claro que el simple estudio de las actitudes no predice la conducta proambiental efectiva y que es fundamental evaluar con mayor profundidad los conocimientos y el contexto en el que ocurre el comportamiento (Álvarez-Suárez, Vega-Marcotey García, 2013; Sandoval, 2012).

Esta concepción plantea el interrogante de si la modificación de las actitudes y la enseñanza de conocimientos ambientales es suficiente para provocar un cambio permanente en el tipo de interacciones que los individuos mantienen con su ambiente, en lo que se ha denominado comportamiento sustentable, dado que la mayoría de currículos escolares en primaria y secundaria han incorporado la educación ambiental, con la idea de que el desarrollo de conocimientos y actitudes pro-ambientales permitirán formar ciudadanos preocupados por el impacto que tiene su comportamiento en el ambiente. No obstante, en concordancia con los cuestionamientos a la visión de actitudes, la evidencia ha mostrado que no existe una relación directa entre el conocimiento, las actitudes y el comportamiento proambiental o sustentable (Gifford, 2014), aun cuando se han identificado aportes positivos a partir del aprendizaje en el contexto de experiencias significativas (ChawlayDerr, 2012).

Algunos estudios realizados para evaluar el comportamiento proambiental en adultos jóvenes universitarios en Colombia (Páramo y Gómez, 1997) como también de la ciudadanía en general (Cortés-Peña, 2011b, 2016b; Garzón-Medina, Barreto y Sandoval-Escobar, 2018; Rodríguez-González y Sandoval-Escobar, 2018), coinciden en señalar la existencia de actitudes positivas hacia el ambiente y un reporte de alto compromiso con el mismo, pero no muestran la relación con el comportamiento proambiental efectivo, como tampoco con el conocimiento sobre las acciones ambientales que resultan más importantes para la preservación del ambiente en diferentes dimensiones. Por otra parte, no se ha establecido si el cursar carreras ambientales influye sobre el desarrollo de mejores comportamientos sustentables y las diferen- 
cias existentes entre las diferentes carreras respecto del cuidado y la valoración del ambiente. Es por ello que la presente investigación se dirigió a responder la pregunta: ¿Cómo es el comportamiento proambiental de los estudiantes universitarios en diferentes disciplinas académicas?

De tal forma, se pretendió identificar las actitudes, creencias, valoraciones, prácticas ambientales y conocimientos ambientales de los estudiantes universitarios colombianos de diferentes carreras de formación y en cincociudades principales del país. En segundo lugar, se establecieron las diferencias existentes entre las carreras de formación y la ciudad en la cual residían los estudiantes, para analizar la influencia de la carrera sobre el comportamiento sustentable y los conocimientos sobre ambiente. Finalmente, se analizó la posible relación existente entre las actitudes, creencias y valores de los estudiantes y las prácticas y el conocimiento ambientales.Estos ejes de indagación permitieron evidenciar las contradicciones existentes entre el decir y el hacer en el comportamiento ambiental de los individuos, mostrando el papel de la educación superior en el desarrollo del comportamiento sustentable de las personas.

\section{Método}

\section{Participantes}

El estudio incluyó 1503 estudiantes de Colombia distribuidos en cinco ciudades principales del país (Bogotá, Cali, Medellín, Barranquilla y Bucaramanga), $52 \%$ de sexo femenino y $48 \%$ de sexo masculino, con un rango de edad entre los 16 y los 50 años, con una concentración mayoritaria delamuestra entre los 16 y los 22 años $(71.9 \%)$. En cuanto al estrato socioeconómico, el $47 \%$ pertenecía al estrato 3, el $22 \%$ al estrato 4 , el $19 \%$ de los estudiantes al estrato 2 , el $7 \%$ al estrato 5 , el $5 \%$ al estrato 1 y solo el $2.7 \%$ al estrato
6. Debido a que la muestra fue seleccionada aleatoriamente de las carreras específicas en las universidades seleccionadas, la variedad de estratos de la muestra corresponde a la distribución de los mismos en dichas instituciones.

\section{Tipo de estudio y muestra}

Se desarrolló un estudio descriptivo correlacional de corte transversal en el cual se incluyeron muestras de cinco ciudades del país elegidas a conveniencia y considerando que cuatro de ellas son las ciudades de Colombia con mayor concentración de población urbana y universitaria, para un total de 1503 estudiantes encuestados. En cada una de las cinco ciudades se seleccionó una proporciónsimilar de la muestra total para evitar que la mayoría quedara concentrada en Bogotá, donde se encuentra el $63 \%$ de la oferta de educación superior de Colombia ${ }^{1}$. Adicionalmente, se consideró únicamente la cantidad de estudiantes matriculados a las áreas de ciencias sociales y humanas, ciencias administrativas y económicas, derecho y afines, ingenierías y matemáticas, así como a ciencias ambientales en el año 2014, de acuerdo con las cifras del Ministerio de Educación Nacional.

Con base en los datos anteriores, la Tabla 1 muestra la cantidad de encuestas realizadas en cada ciudad y las carreras a las que pertenecían los estudiantes seleccionados. La selección final mantuvo muy similar la cantidad de encuestas por ciudad y la proporción de estudiantes correspondiente a cada uno de los campos de conocimiento de interés para la presente investigación; no obstante, en Bogotá y en Barranquilla estas proporciones variaron en virtud de los permisos concedidos para acceder a los grupos de las carreras.

Esta es la razón por la cual en general las muestras de carreras pertenecientes a ciencias económicas y administrativas, así como a las carreras de ciencias sociales, son mayores que

\footnotetext{
'http://www.mineducacion.gov.co/sistemasdeinformacion/1735/articles-212352_bogota.pdf
} 
las demás muestras. Se escogieron estudiantes de todos los semestres de las carreras consideradas, debido a que la cantidad de semestres cursados por el estudiante encuestadono fue una variable de selección muestral.Se trató de una muestra probabilística con selección aletoria en cada universidad, la cual cuenta con una variable de diseño cualitativa con una probabilidad de incidencia de .5 , un $95 \%$ de confianza y un error del $2.5 \%$ (ver Tabla 1$)$.

Tabla 1. Distribución de estudiantes seleccionados por ciudad.

\begin{tabular}{cccccccc} 
& CEA & CEI & CS & CA & CJ & Total & Error \% \\
\cline { 2 - 7 } Bogotá & 102 & 50 & 48 & 50 & 50 & 300 & 5.6 \\
Barranquilla & 60 & 60 & 66 & 56 & 60 & 302 & 5.6 \\
Bucaramanga & 59 & 60 & 60 & 61 & 60 & 300 & 5.6 \\
Medellín & 84 & 18 & 117 & 29 & 53 & 301 & 5.6 \\
Cali & 60 & 60 & 60 & 60 & 60 & 300 & 5.6 \\
Total & 365 & 248 & 351 & 256 & 283 & 1503 & 2.5
\end{tabular}

CEA: Ciencias Económicas y Administrativas. / CEI: Ciencias Exactas e Ingenierías. / CS: Ciencias Sociales. / CA: Ciencias Ambientales. / CJ: Ciencias Jurídicas.

\section{Instrumentos}

Seaplicaron dos cuestionarios cuyo objetivo fue medir el comportamiento proambiental en sus diferentes dimensiones. El primer instrumento fue el Inventario de Comportamiento Proambiental(ICP) desarrollado por Sandoval y Barreto (2014). El segundo cuestionario, el Test de Conocimiento Ambiental (TCA) fue desarrollado, piloteado y ajustado para propósitos de la presente investigación.

\section{Inventario de Comportamiento Proambiental (ICP).}

Este instrumentoincluye ítems del cuestionario de Paternina (2007), quien adaptó a su vez la escala de González-López (2002) y contiene otros ítems desarrollados por Sandoval y Barreto (2014) para la evaluación del comportamiento proambiental de las personas en Colombia. El cuestionario de González-López (2002) contiene un total de siete escalas basadas en algunos de los cuestionarios más empleados para medir actitudes ambientales. Entre estos se encuentran el Índice de Control Interno de Conducta Ambiental de Smith-Sebasto y Fortner (1994), el cuestionario de Dunlap et al. (2000), denominado Nuevo Paradigma Ecológico (NPE), y la Escala de Valores (SVS) de Schwartz (1994). Las escalas finales del cuestionario empleado por González-López (2002) fueron: a) Escala del Nuevo Paradigma Ecológico (NPE), b) Escala de Conciencia General de las Consecuencias Ambientales (CGC), c) Escala General de Conducta Ecológica (COGE), d) Escala de Negación de la Obligación Ambiental (NOA), e) Escala de Normas Personales Ambientales (NPA), f) Escala de Control de Conducta Ambiental (ECA) y g) Escala de Valores (EV).

Por su parte, el cuestionario de Sandoval y Barreto (2014) empleado en Colombia para investigar el comportamiento proambiental en hogares de la ciudad de Bogotá mostró una alta fiabilidad (Alfa de Cronbach de .883) e incluyó 67 ítems que evaluaron: a) creencias 
ambientales (15 ítems), b) normas subjetivas ambientales ( 25 ítems) y c) reporte de acciones proambientales y antiambientales (27 ítems). Los ítems para medir creencias y normas subjetivas se calificaron en escalas Likert de 6 grados, mientras que los ítems desarrollados para medir acciones proambientales y antiambientales se calificaron a través de una escala de frecuencia con 5 grados. Las creencias se calificaron a través de una escala de acuerdo con los siguientes grados: Total Desacuerdo (TD), Muy en Desacuerdo (MYD), Moderado Desacuerdo (MD), Moderado Acuerdo (MA), Muy de Acuerdo (MYA), Total Acuerdo (TA). Las normas subjetivas y reglas se evaluaron a través de una escala de importancia con los siguientes grados: Sin importancia (SI), Muy poco importante (MYPI), Poco importante (PI), Algo Importante (AI), Muy Importante (MYI), Totalmente Importante (TI). Finalmente, las acciones proambientales se evaluaron a través de una escala de frecuencia con los siguientes niveles: Nunca (NU), Rara vez (RV), A veces (AV) Frecuentemente (FR), Siempre (SPR). En el presente estudio se obtuvo un Alfa de Cronbach de .864, valor muy similar al del estudio original de Sandoval y Barreto (2014).

\section{Test de Conocimiento Ambiental (TCA).}

El TCA es un instrumento diseñado para propósitos de la presente investigación y tuvo como objetivo evaluar los conocimientos en ambiente que poseen los estudiantes universitarios en Colombia. Se construyó con base en algunos de los criterios establecidos en las pruebas Saber 11 respecto de biodiversidad y ecología, así como en los conocimientos que deben serrequeridos por parte de la ciudadanía para alcanzar las competencias definidas por el Ministerio de Educación de Colombia para los objetivos de desarrollo sostenible del país. Originalmente la prueba contenía 19 ítems organizados alrededor de las siguientes áreas: a) Biodiversidad y sostenibilidad, b) Manejo de residuos sólidos urbanos, c) causas de las problemáticas ambientales y d) consumo sustentable. La prueba original fue sometida a dos procedimientos durante la fase de pilotaje. En primera instancia se analizó su validez de contenido a través de los conceptos de tres jueces expertos en el campo de la psicometría y de la construcción de test en educación. La calificación final de los jueces fue promediada y se ajustaron los ítems que habían recibido las calificaciones inferiores a 5. De igual modo, de acuerdo con las observaciones de los jueces, se corrigieron algunos términos empleados en los enunciados de los ítems. En una etapa posterior, se aplicó el instrumento a 92 estudiantes universitarios de las carreras de Psicología, Ingeniería y Administración de una universidad bogotana. Los resultados obtenidos fueron analizados a partir de la teoría clásica de los test y también desde la visión alternativa de la Teoría de Respuesta al Ítem (IRT). A partir de los procedimientos realizados durante el pilotaje del TCA, se determinó la eliminación de dos de los ítems originales, de modo que la prueba aplicada a los 1503 estudiantes universitarios del país incluyó 16 ítems, 14 de ellos de selección múltiple con 4 distractores,un ítem de correspondencia con 6 elementos y un ítem de falso-verdadero con 4 elementos.

\section{Procedimiento}

En primera instancia, se solicitó el permiso de diferentes universidades en las 5 ciudades seleccionadas. Una vez que se contaba con la posibilidad de acceso a las carreras particulares, se procedió al desarrollo de consentimientos informados a cada uno de los estudiantes, en el cual se establecían los objetivos del estudio y el uso académico de la información. Los cuestionarios fueron de autoaplicación grupal con papel y lápiz, con una duración promedio de 20 minutos. Los datos fueron procesados empleando el programa SPSS-20. 


\section{Resultados}

\section{Resultados generales del Inventario de Comportamiento Proambiental}

Los resultados muestran que los estudiantesencuestadosposeen actitudes y creencias ambientales positivas en la mayoría de las dimensiones, como también una valoración de alternativas de sellos ecológicos, transportes, productos y marcas amigables con el ambiente. Se encuentra una gran dispersión en la frecuencia con la cual las personas realizan algunas acciones proambientales, pero en la mayoría de los casos reportan diferentes prácticas proambientales.

De igual modo, se encontraron diferencias en las creencias, valoración y acciones ambientales de los estudiantes encuestados en las diferentes ciudades y en las diferentes carreras. En cuanto a los resultados del ICP de acuerdo con la carrera, se encontraron diferencias en ítems específicos en cada una de las escalas. En la escala de creencias acerca del ambiente, la Tabla 2 muestra una diferencia de medias positiva y significativa (Diferencia de Medias Carrera I- Carrera J, $p$ $<.05)$ de ciencias sociales y ciencias jurídicas en comparación a las ciencias ambientales en cuanto a la intención de comprar productos recomendados por organizaciones ambientalistas. También los estudiantes de las carreras correspondientes a ciencias económicas y administrativas estarían más dispuestos que los estudiantes de ciencias ambientales a comprar productos más costosos que protegieran el ambiente. Asimismo, los estudiantes de ciencias económico-administrativas, así como de las ingenierías aventajan a los de ciencias ambientales y de ciencias jurídicas en la creencia de que los seres humanos están abusando en exceso del medio ambiente. Los estudiantes de ciencias económicas y administrativas están más dispuestos que los de ciencias exactas e ingenierías a participar en actividades en pro del ambiente, mientras que los estudiantes de ciencias jurídicas poseen una mayor intención que los de ciencias ambientales de comprar el siguiente mes productos ecológicos. 
Tabla 2. Diferencia de medias significativas en las creencias ambientales de acuerdo con la carrera.

\begin{tabular}{|c|c|c|c|c|c|}
\hline \multicolumn{3}{|c|}{ Escala de Creencias y Carreras } & \multirow{2}{*}{$\begin{array}{l}\text { Diferencia } \\
\text { de Medias } \\
\quad(\mathrm{I}-\mathrm{J})\end{array}$} & \multirow{2}{*}{$\begin{array}{l}\text { Error } \\
\text { Típico }\end{array}$} & \multirow{2}{*}{$\begin{array}{l}\text { Sig. } \\
*\end{array}$} \\
\hline & Carrera I & Carrera J & & & \\
\hline \multirow{2}{*}{$\begin{array}{l}\text { Compraría algún } \\
\text { producto o servicio } \\
\text { ambiental si una } \\
\text { organización ecológica } \\
\text { fuera la que lo } \\
\text { recomendara. }\end{array}$} & Ciencias Sociales & $\begin{array}{c}\text { Ciencias } \\
\text { Ambientales }\end{array}$ & $.297^{*}$ & .109 & .050 \\
\hline & Ciencias Jurídicas & $\begin{array}{c}\text { Ciencias } \\
\text { Ambientales }\end{array}$ & $.388 *$ & .119 & .010 \\
\hline \multirow{3}{*}{$\begin{array}{l}\text { Los seres humanos están } \\
\text { abusando en exceso del } \\
\text { medio ambiente. }\end{array}$} & $\begin{array}{c}\text { Ciencias } \\
\text { Económicas y }\end{array}$ & $\begin{array}{c}\text { Ciencias } \\
\text { Ambientales } \\
\end{array}$ & $.465^{*}$ & .121 & .001 \\
\hline & Administrativas & Ciencias Jurídicas & .329 & .120 & .050 \\
\hline & $\begin{array}{c}\text { Ciencias Exactas e } \\
\text { Ingenierías }\end{array}$ & $\begin{array}{c}\text { Ciencias } \\
\text { Ambientales }\end{array}$ & $.374^{*}$ & .135 & .045 \\
\hline \multirow{4}{*}{$\begin{array}{l}\text { Compraría algún } \\
\text { producto favorable al } \\
\text { ambiente aunque fuera } \\
\text { mucho más costoso } \\
\text { que los productos } \\
\text { no amigables con el } \\
\text { ambiente. }\end{array}$} & $\begin{array}{c}\text { Ciencias } \\
\text { Económicas y }\end{array}$ & $\begin{array}{c}\text { Ciencias Exactas } \\
\text { E Ingenierías } \\
\end{array}$ & $.325^{*}$ & .101 & .012 \\
\hline & Administrativas & Ciencias Sociales & $.308^{*}$ & .098 & .015 \\
\hline & $\begin{array}{c}\text { Ciencias Exactas e } \\
\text { Ingenierías }\end{array}$ & $\begin{array}{c}\text { Ciencias } \\
\text { Económicas y } \\
\text { Administrativas } \\
\end{array}$ & $-.325^{*}$ & .101 & .012 \\
\hline & Ciencias Sociales & $\begin{array}{c}\text { Ciencias } \\
\text { Económicas y } \\
\text { Administrativas } \\
\end{array}$ & $-.308^{*}$ & .098 & .015 \\
\hline $\begin{array}{l}\text { Estoy dispuesto/a } \\
\text { realizar diferentes } \\
\text { actividades para proteger } \\
\text { el medio ambiente y } \\
\text { evitar su deterioro. }\end{array}$ & $\begin{array}{c}\text { Ciencias } \\
\text { Económicas y } \\
\text { Administrativas }\end{array}$ & $\begin{array}{l}\text { Ciencias Exactas e } \\
\text { Ingenierías }\end{array}$ & $.332 *$ & .114 & .031 \\
\hline $\begin{array}{l}\text { Con seguridad el } \\
\text { próximo mes comprare } \\
\text { productos ecológicos. }\end{array}$ & Ciencias Jurídicas & $\begin{array}{c}\text { Ciencias } \\
\text { Ambientales }\end{array}$ & $.359 *$ & .116 & .017 \\
\hline
\end{tabular}

En cuanto a la valoración de normas y acciones ambientales e importancia concedida a diferentes comportamientos relacionados con el ambiente, se observaron diferencias entre medias significativas y positivas (Diferencia $\mathrm{I}-\mathrm{J}, p<.05)$ entre algunas carreras $\mathrm{y}$ en algunos ítems particulares. Entre otros, los resultados muestran que los estudiantes de ciencias jurídicas muestran una tendencia a valorar más acciones, organismos, inter- venciones y preocupaciones ambientales, en comparación a los de las demás carreras. Los estudiantes de ciencias ambientales se preocupan más por consumir productos que tengan sellos verdes, al mismo tiempo que valoran más que los de ciencias exactas e ingenierías y que los de ciencias sociales el hecho de que existan organismos internacionales que velan por el cumplimiento de normas ambientales y por el desarrollo de programas para estimular 
el cuidado del medio ambiente. En este último aspecto también aventajan a los estudiantes de ciencias económicas y administrativas.

A pesar de lo expresado anteriormente, los estudiantes de ciencias económicas y administrativas, así como los estudiantes de ciencias jurídicas, muestran una mayor tendencia a valorar positivamente acciones antiambientales tales como consumir productos fumigados con plaguicidas, comprar frutas o verduras empacadas y comprar un producto eficiente aunque no sea ecológico.

En cuanto a las acciones ambientales y antiambientales, existen diferencias en la frecuencia reportada de ejecución de tales actos dependiendo de la carrera que estudian las personas $(p<.05)$. Entre otros resultados, se aprecia que con mayor frecuencia los estudiantes de ciencias exactas e ingenierías compran productos orgánicos para el consumo de la familia y artículos que contengan sellos ambientales, en comparación con los estudiantes de económicas y administrativas, ciencias sociales, ciencias jurídicas y ciencias ambientales. Los estudiantes de ciencias exactas e ingenierías reportan el uso más frecuente de detergentes biodegradables, como también diversos comportamientos de ahorro de energía en comparación con los estudiantes de ciencias ambientales, ciencias jurídicas y ciencias económicas.

En cuanto al reporte de acciones antiambientales, los estudiantes de ciencias exactas e ingenierías más frecuentemente utilizan productos abrasivos en la cocina, en comparación con los estudiantes de ciencias ambientales y ciencias jurídicas; los estu- diantes de ciencias económicas y administrativas desechan el aceite por el lavaplatos con mayor frecuencia que los de ciencias ambientales y ciencias jurídicas; los de ciencias jurídicas usan más frecuentemente insecticidas que los de ciencias ambientales y que los de ciencias económicas y administrativas.Finalmente, los estudiantes de ciencias exactas e ingenierías, como también los de ciencias sociales,con mayor frecuencia desperdician agua en la ducha y en el cepillado de dientes, en comparación con ciencias económicas y administrativas, así como ciencias ambientales. En general, los resultados muestran que al mismo tiempo que las personas realizan comportamientos proambientales, también desempeñan diversos comportamientos antiambientales.

\section{Resultados del Test de Comportamiento Ambiental (TCA): Conocimientos sobre ambiente}

A través del TCA se evaluó el conocimiento ambiental de los estudiantes en las cinco ciudades seleccionadas. En general se encontró un puntaje promedio de 12 puntos sobre 24 posibles $(50 \%)$, lo que significa que reprobaron el test.En relación con los conocimientos ambientales medidos a través de cada uno de los ítems del TCA, se observa que la gran mayoría de las personas (porcentajes superiores al $70 \%$ ) sabe qué es la biodiversidad, cuáles son sus amenazas, en qué consiste un producto ecológico y conocenla capacidad contaminante de diferentes materiales (Tabla 3 ). 
Tabla 3. Porcentaje de aciertos en el Test de Conocimiento Ambiental (TCA).

\begin{tabular}{|c|c|c|}
\hline Conocimientos & Correcto & Incorrecto \\
\hline Qué es la biodiversidad & 81.4 & 18.6 \\
\hline En que consiste la lluvia ácida & 40.7 & 59.3 \\
\hline Qué es un contaminante indirecto de la atmósfera & 40.7 & 59.3 \\
\hline A qué hace referencia el reciclaje & 43.5 & 56.5 \\
\hline Gasto de energía de diferentes electrodomésticos & 56.6 & 43.4 \\
\hline Amenazas a la biodiversidad & 74.1 & 25.9 \\
\hline $\begin{array}{l}\text { Qué significa el término preservación en los temas } \\
\text { ambientales }\end{array}$ & 62.5 & 37.5 \\
\hline $\begin{array}{l}\text { Colores que identifican los contenedores principales del } \\
\text { reciclaje }\end{array}$ & 63.7 & 36.3 \\
\hline Color de los contenedores para envases de plástico & 18.8 & 81.2 \\
\hline Qué es la composta & 52.9 & 47.1 \\
\hline $\begin{array}{l}\text { Efectos de los detergentes biodegradables en la } \\
\text { contaminación aguas }\end{array}$ & 66.4 & 33.6 \\
\hline Reciclaje de los termoplásticos & 62.1 & 37.9 \\
\hline Funciones de la ecología como disciplina & 62.9 & 37.1 \\
\hline $\begin{array}{l}\text { Tipos de selección y técnicas desarrolladas por los } \\
\text { humanos }\end{array}$ & 33.9 & 66.1 \\
\hline $\begin{array}{l}\text { Problemas recolección y disposición de residuos sólidos } \\
\text { urbanos }\end{array}$ & 23.7 & 76.3 \\
\hline Quema de combustibles fósiles & 28.0 & 72.0 \\
\hline Uso de fertilizantes y plaguicidas para los cultivos & 58.0 & 42.0 \\
\hline $\begin{array}{l}\text { Vertimientos no controlados de residuos líquidos } \\
\text { industriales }\end{array}$ & 47.4 & 52.6 \\
\hline Desaparición de la vegetación por la acción humana & 55.8 & 44.2 \\
\hline Vertimiento de sustancias refrigerantes & 58.0 & 42.0 \\
\hline Qué es un producto orgánico & 31.3 & 68.7 \\
\hline Qué es un producto natural & 22.0 & 78.0 \\
\hline Qué es un producto ecológico & 77.3 & 22.7 \\
\hline Capacidad contaminante de diferentes materiales & 71.1 & 28.9 \\
\hline
\end{tabular}

Por otra parte, una gran proporción de personas no saben cuál es el color de los contenedores para envases de plástico $(81 \%)$, qué es un producto natural $(78 \%)$, los problemas existentes con la recolección y disposición de los residuos sólidos urbanos (76\%), la quema de combustibles fósiles (72\%), qué es un producto orgánico (68\%) y los tipos de selección a través de técnicas desarrolladas por los humanos (66 \%). En cuanto a las carreras, se observaron pocas diferencias significativas en el conocimiento ambiental de acuerdo con la carrera que estudiaba el entrevistado, dado que el promedio fue muy similar en todas las carreras $(F=1.243 ; p=.290)$. 


\section{Análisis multivariado}

Se realizó un análisis de correlación canónica y un análisis factorial para identificar las relaciones entre las variables evaluadas en los cuestionarios. El valor de la correlación canónica entre estos pares de subconjuntos de ítems fuealta en todos los casos y mostró una importante cantidad de varianza compartida, sobretodoentre la escala de creencias y la de importancia de aspectos ambientales. En los demás pares el cuadrado de la correlación se aproximaal $30 \%$. En general, las correlaciones canónicas indican que existen al menos 6 ítems de la escala de creencias que predicen las respuestas en la escala de importancia de aspectos ambientales, las acciones reportadas y los conocimientos acerca del ambiente medidos en el TCA. Lo mismo sucede con 13 ítems de la subescala de importancia y con 7 acciones ambientales. Solamente 6 ítems del TCA predicen otras respuestas en el ICP.

En cuanto al análisis factorial,para identificar la estructura interna de las pruebas aplicadas y analizar las relaciones existentes entre estos factores empleando el método de extracción a través del análisis de componentes principales, se exploraron los factores conceptuales del ICP asociados con el modelo de Ajzen (2002), clasificando los ítems en los siguientes constructos: a) creencias conductuales, b) creencias de control, c) creencias normativas, d) normas subjetivas, e) intención y f) acción.

El constructo creencias conductuales arrojó doscomponentesexplicando el mayor porcentaje de varianza. Estos componentes se denominaron Creencias de Consumo Ecológico y Creencias de Consumo Tecnológico, incluyendo los ítems relacionados con el consumo en pro de la ecología y aquellos vinculados con el uso de aparatos tecnológicos, respectivamente. Para el caso de las creencias de control, se encontraron dos componentes: Creencias Pesimistas y Creencias Optimistas. La Tabla 4 muestra el análisis final para la prueba ICP. Es claro que el conjunto de ítems denominado Creencias de Consumo Ecológico, correspondientes al constructocreencias conductuales, forma parte del componente 1, al que también pertenecen las creencias normativas, las creencias de control pesimistas, la intención y las normas subjetivas. Por otra parte, las creencias conductuales denominadas de Consumo Tecnológico, las creencias de control optimistas y las acciones comportamentales forman parte del componente 2 .

Tabla 4. Factores identificados en el Inventario de Comportamiento Proambiental (ICP).

\begin{tabular}{lccc} 
& & \multicolumn{2}{c}{ Componente } \\
& Factores identificados & 1 & 2 \\
\hline \multirow{2}{*}{ Creencias conductuales } & Creencias consumo ecológico & .861 & .100 \\
& Creencias consumo tecnológico & -.203 & .668 \\
\cline { 2 - 3 } Creencias de control & Creencias normativas & .880 & -.161 \\
& Creencias pesimistas & .795 & -.269 \\
& Creencias optimistas & .344 & .671 \\
& Intención & .810 & .015 \\
& Norma subjetiva & .489 & .429 \\
& Acciones proambientales & -.066 & .713 \\
\hline
\end{tabular}


En el TCA se exploraron los factores con los que se construyó la prueba y que se describieron en la sección de instrumentos. Estos factores fueron: a) Conocimientos sobre biodiversidad y sostenibilidad, b) Manejo de residuos sólidos urbanos (RSU), c) Causas de problemáticas ambientales y d) Consumo sustentable. El análisis factorial mostró la existencia de otros factores que describieron mejor los resultados y que se observan en la Tabla 5.

Tabla 5. Análisis de componentes del Test de Comportamiento Ambiental (TCA).

\begin{tabular}{cccc} 
Factores del TCA & \multicolumn{3}{c}{ Componente } \\
\hline Conocimientos Sobre Biodiversidad y Sostenibilidad & 1 & 2 & 3 \\
\hline ECOBIOAP & .609 & .249 & -.082 \\
CONCECOBIO & -.316 & .521 & .046 \\
Conocimientos En Manejo General De Rsu & & & \\
\hline RSU & .616 & .189 & -.064 \\
PROCRSU & -.131 & .675 & -.144 \\
Causas De Problemáticas Ambientales & & & \\
\hline OTRACONTAMINACION & .466 & .529 & -.062 \\
CONTAMINATMOSFER & -.412 & .455 & -.106 \\
EFECTOSAMBIENTE & .004 & .069 & .878 \\
Consumo Sustentable & & & .147 \\
\hline CONSUMOSUSTENTABLE & .698 & -.067 & .420 \\
CONSUMORGANICOS & -.086 & .323 & \\
\hline
\end{tabular}

Se realizó un análisis multivariado con un modelo lineal con el fin de evaluar la relación entre los factores identificados. Las covarianzas entre los factores del ICP y del TCA resultaron altas, hecho que ya se había evidenciado en el análisis de correlaciones canónicas. Los conocimientos aplicados y especializados sobre ambiente en relación con biodiversidad, el conocimiento sobre contaminación atmosférica, los conocimientos sobre consumo sustentable, así como los conocimientos en procesos de reciclaje, se relacionan de forma importante con casi todos los factores del ICP, incluyendo el reporte de acciones proambientales. Los conocimientos sobre consumo de productos orgánicos covaríancon las creencias acerca del consumo ecológico, las normas subjetivas y las creencias pesimistas, pero no con las acciones ambientales. Por otra parte, el conocimiento general sobre el manejo de residuos sólidos urbanos y sobre efectos de fuentes de contaminación no atmosférica sobre el ambiente no covarían signi- ficativamente con las acciones proambientales, ni con los demás factores del ICP.

\section{Discusión}

El estudio descriptivo correlacional desarrollado en cinco ciudades de Colombia y con estudiantes de diferentes carreras muestra con claridad que existen diferentes paradojas en relación con el comportamiento ambiental. Se esperaba que los estudiantes universitarios, quienes han alcanzado el máximo nivel educativo en el país, mostraran excelentes actitudes, conocimientos ambientales y acciones signficativas en pro del ambiente. Igualmente se suponía que los estudiantes de aquellas carreras profesionales más relacionadas con el ambiente, mostrarían mejores conocimientos y acciones proambientales. Los resultados muestran que no fue así.

En particular, respecto de los conocimientos acerca de ambiente, en los resultados 
descriptivos el promedio de la prueba fue de 12 puntos, lo que significa que la mayoría de los estudiantes reprobaron el test. Como se indicó previamente, quizá el resultado más sorprendente encontrado a través del presente estudio es que no se observaron diferencias significativas entre las carreras respecto a las creencias, valoraciones, acciones y conocimientos ambientales.Más aún, en el análisis de ítems específicos los estudiantes de ciencias ambientales ocuparon lugares inferiores a los que estudiaban ciencias económicas y administrativas, ciencias jurídicas y ciencias exactas e ingenierías. Tampoco realizaban más acciones proambientales que los demás y realizaban diversas acciones antiambientalescomotambién lo hacían los estudiantes de todas las carreras investigadas.

El análisis multivariado mostró que el modelo teórico en el cual se basó la construcción del ICP y los instrumentos de los que se seleccionaron ítems para su conformación, aparentemente posee un gran poder predictivo respecto al reporte verbal de acciones ambientales, pero que el TCA-prueba construida específicamente para esta investigación-covaría con casi todos los factores del ICP pero no predice causalmente las acciones reportadas por los estudiantes. Aun cuando puede existir covariación entre los conocimientos sobre ambiente y diferentes aspectos relacionados con las actitudes ambientales, cuando se prueba su capacidad para predecir las acciones ambientales reportadas, el conocimiento de los estudiantes no posee este poder predictivo. Por el contrario, el conocimiento sobre consumo sustentable y la preocupación ambiental implican que las personas hagan menos acciones ambientalmente relevantes. Igualmente, se encontró una relación entre los diferentes factores identificados en el ICP respecto de las acciones ambientales reportadas.

Como en otros estudios realizados en el mundo, los universitarios de Colombia muestran creencias, normas e intenciones favorables hacia el ambiente;no obstante, evidencian más desacuerdos cuando se trata de invertir más dinero en artículos o compañías amigables con el ambiente, como también cuando se trata de renunciar a las comodidades propias de la vida urbana como son el uso de celular y del carro para transportarse. Igualmente, en algún sentido minimizan su sentido de control de los resultados ambientales debido a que creen que es el gobierno el que tiene que crear más programas de educación ambiental y que su contribución a los problemas ambientales es moderada. Al mismo tiempo, rechazan la intervención humana para modificar el ambiente y creen que es importante que las universidades, el gobierno y otras organizaciones investiguen sobre el ambiente. Este resultado es similar al encontrado por Páramo et al. (2015) en Latinoamérica con una muestra de 9 países en los que se investigó la percepción de las personas respecto a su ambiente local, nacional y global, así como la responsabilidad atribuida a diferentes agentes en cuanto a los resultados ambientales. Como en el presente estudio, se estableció que los ciudadanos atribuyen la responsabilidad principalmente al gobierno y a instancias internacionales, dejando su papel en un lugar secundario. Este resultado, discuten la misma línea de Uzell (2000), Uzell, Pol y Badenas (2002), como también de Vlek y Steg (2007) puede obedecer a que los ítems seleccionados para el ICP comprenden preguntas acerca del ambiente en general, sin particularizar al contexto local en el cual se desenvuelven los estudiantes. Cuando ese es el caso, las personas suelen ser más propositivas en su atribución de responsabilidad acerca de las problemáticas ambientales.

El reporte de acciones ambientales en los estudiantes universitarios presenta un perfil controversial. Aunque una gran cantidad de personas examinan los componentes de los productos, tratan de ahorrar energía eléctrica, rara vez o nunca desechan el aceite y los restos líquidos por el desagüe y compran frecuentemente verduras orgánicas; aproximadamente la mitad de los estudiantes no reutilizan el agua de la lavadora, con frecuencia se dan 
duchas de 10 minutos, no enjuagan los vasos de lácteos para que puedan ser reutilizados, emplean con frecuencia artículos que generan contaminación atmosférica, amenazan la biodiversidad de las aguas, botan las pilas a la basura normal y frecuentemente arruinan el papel que desechan de manera que no puede ser reciclado. Lo anterior significa que poseen un gran conjunto de prácticas antiambientales, las cuales reportan de forma abierta. El estudio demostró que dichas prácticas se presentan en algunas carreras más que en otras, pero los resultados fueron sorprendentes en la medida en que los estudiantes de ciencias ambientales reportan diversas acciones antiambientales, cuando se espera que su mayor conocimiento respecto a las temáticas ambientales correlacione con el comportamiento sustentable.

Al respecto, el conocimiento ambiental evaluado a través del TCA mostró una media de 50 sobre 100 , esto significa que los estudiantes evaluadosno aprobaron la prueba, aun cuando en las ciudades de Bucaramanga y Cali las medias fueron un poco mayores. En general, el mayor desconocimiento se presenta en temas de reciclaje, combustibles fósiles, productos orgánicos y procesos de selección artificial, pero una gran proporción de personas no sabe cuáles son los efectos de diversas sustancias contaminantes, las amenazas para la vegetación, los efectos del uso de fertilizantes y pesticidas, las formas de contaminación a través de residuos líquidos industriales, los contaminantes de la atmósfera, en qué consiste la lluvia ácida y el gasto de energía de los diferentes electrodomésticos.

El TCA evalúa los conocimientos básicos que cualquier ciudadano del común debería tener acerca de aquellos factores que amenazan la calidad del ambiente y que pueden incidir en la ejecución de acciones con resultados ambientales distintos. Si se toma en cuenta que los estudiantes no conocen aproximadamente la mitad de aspectos ambientales importantes para lograr este comportamiento susten- table, debe suponerse que no se han generado procesos educativos al interior de los hogares, en las ciudades y dentro de las universidades para formar una cultura proambiental en los adultos jóvenes. Esta cultura proambiental es mucho más que los saberes ambientales.

Infortunadamente las relaciones existentes en la literatura entre el conocimiento y las actitudes hacia el ambiente, así como entre estos y las accionesproambientales poseen evidencias a favor y en contra (Corral-Verdugo y Pinherio, 2004, 2009; Corral-Verdugo, 2010; Corral-Verdugo et al., 2012; Corral-Verdugo, 2012a, 2012b; Sandoval, 2012). No obstante, esta medición se ha desarrollado a partir de cuestionarios generales fundamentados en distintos modelos teóricos de actitudes, creencias, valores e influencia social, sin considerar los valiosos elementos contextuales que afectan significativamente las relaciones entre los individuos y su entorno (Amérigo, 2006; Berenguer y Corraliza, 2000; GarcíaMira, 2009). La Figura 1 permite observar las relaciones identificadas en el presente estudio y que concuerdan con los hallazgos de otras investigaciones en Latinoamérica.

A pesar de las inconsistencias encontradas en la literatura respecto a las variables que mejor predicen las acciones ambientales, es innegable que los estudios se han basado en escalas que han sido aplicadas en diferentes países e idiomas, y que a partir de los modelos multidimensionales de la actitud, algunos cuestionarios han predicho actos proambientales particulares. El cuestionario ICP empleado en este estudio se inspiró en otras escalas basadas en modelos de actitudes, especialmente en los modelos de Ajzen y Fishbein (2005), Fishbein y Ajzen (2011), así como de la Teoría de la Conducta Planeada de Ajzen $(1991,2002)$ los cuales establecen la importancia de evaluar de forma específica las creencias, normas y valoraciones, con el fin de lograr una mejor predicción acerca de las acciones que reportan los individuos. 


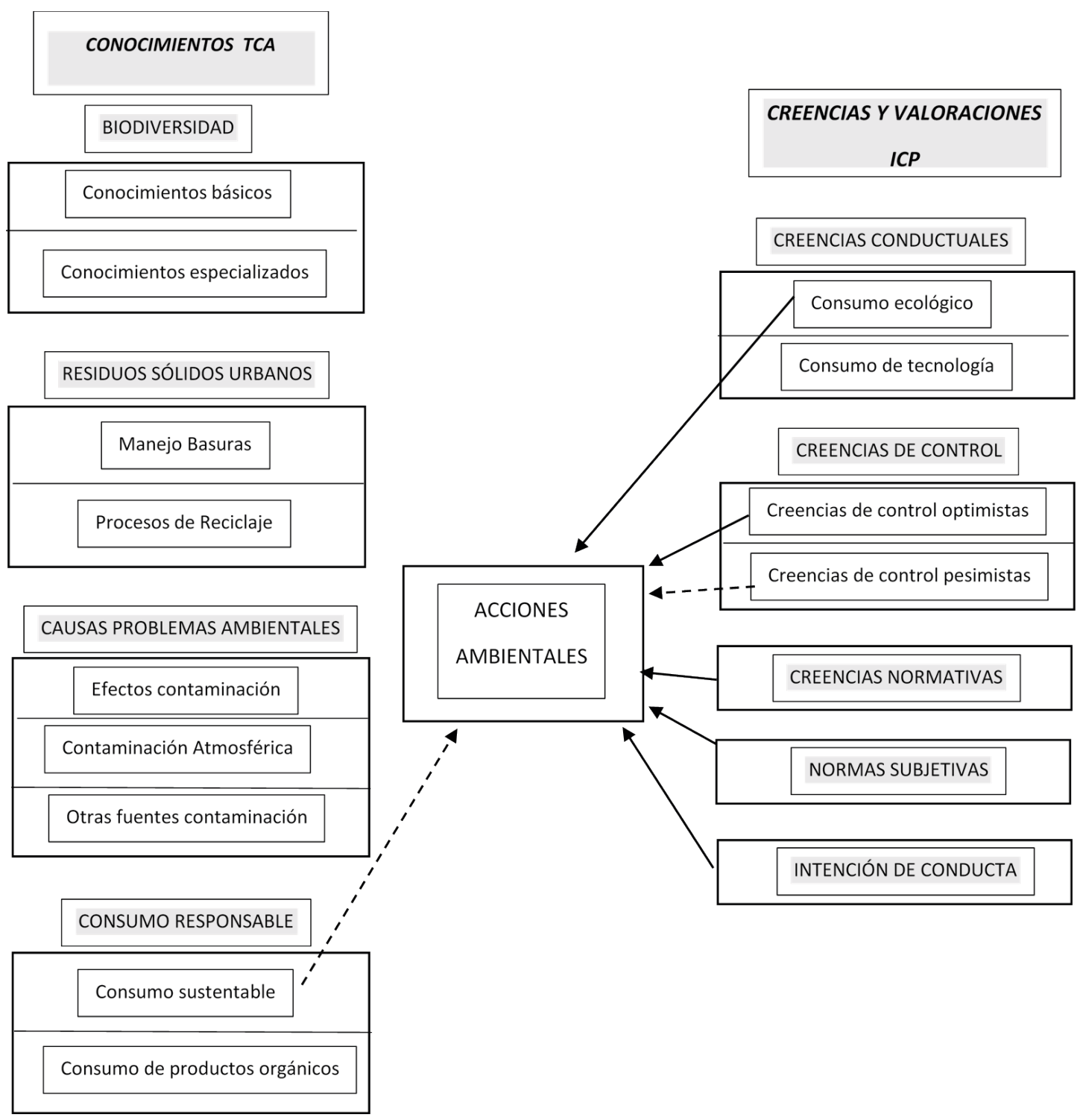

Figura 1. Relación entre las variables consideradas en el estudio.

Los hallazgos esquematizados en la Figura 1 concuerdan con la teoría de Ajzen (2002), la cual plantea que las creencias, actitudes, intenciones y comportamientos de los individuos se relacionan con el proceso de decisión de los individuos, estableciendo que no existe una relación causal entre la actitud y el comportamiento efectivo. Se postula que la influencia de las creencias y actitudes se dan sobre la intención y luego de ello, las intenciones pueden afectar las acciones efectivas. En el TCP las expectativas acerca del comportamiento de otros individuos en el contexto social se observan a partir de las normas subjetivas y el control conductual percibido, los cuales se relacionan significativamente con las creencias y pueden predecir las intenciones. Las investigaciones sobre predictibilidad de las actitudes y creencias evidencian que en muchas ocasiones las personas experimentan dificultades al estimar cómo actuarían en determinadas circunstancias y por ende en muchos casos se alejan de la conducta efectiva (Gifford, 2014; Gifford y Sussman, 2012).

Los resultados aparentemente podrían interpretarse como evidencia en contra de la 
importancia de la educación ambiental para el logro de un comportamiento proambiental; sin embargo, varios puntos impiden extraer esta apresurada conclusión. En primer lugar, es posible que las creencias, valoraciones y normas relacionadas con el ambiente se relacionen con una dimensión de la educación ambiental. En virtud de los resultados obtenidos, esta dimensión es importante dado que predice de forma consistente el reporte de acciones ambientales (Medina y Páramo, 2014; Crissien-Borrero, Cortés-Peña y Herrera-Mendoza, 2016). En segundo lugar, el hecho de que el promedio de la prueba de conocimientos fuera reprobatorio precisamente significa que en el caso particular de este estudio, no toda la información consolidada (aquella que conforma el factor) acerca del ambiente se relaciona con las prácticas reportadas, pero que por separado, el conocimiento de aspectos particulares mostró correlaciones con diferentes aspectos del ICP y con algunas acciones ambientales. Este fue el caso de los ítems relacionados con las amenazas a la biodiversidad, los conocimientos sobre ecología, el conocimiento de problemas de recolección y disposición de residuos sólidos urbanos y la diferenciación de productos orgánicos-naturales. Así por ejemplo, el conocer más sobre biodiversidad y sobre productos ecológicos correlaciona negativamente con las creencias ambientales, mientras que saber de reciclaje y manejo de RSU correlaciona positivamente con las mismas. Estos mismos aspectos correlacionan positivamente todos con las valoraciones de reglas y normas ambientales, así como con las acciones reportadas.

Al considerar el par de acciones ambientales y el TCA, las correlaciones canónicas muestran que tomando en cuenta toda la prueba de conocimientos, se observan altas correlaciones con acciones para preparar los desechos para el reciclaje y usar responsablemente servicios públicos, al mismo tiempo que se observan correlaciones negativas con prácticas antiambientales como botar las pilas a la basura. A pesar de esto, se observan también correlaciones positivas con acciones antiambientales como botar restos líquidos de comida por las tuberías del desagüe y dejar el televisor encendido cuando no se está utilizando. Lo anterior implica que no se puede concluir que los conocimientos sobre ambiente son innecesarios (Cortés-Peña, 2011a; Cortés-Peña, 2016b; Sapiainsy Ugarte, 2017a; Sapiainsy Ugarte, 2017b).

Los resultados del presente estudio son un desafío para las instituciones universitarias y para el gobierno. En una gran cantidad de aspectos del comportamiento, la educación ambiental no muestra los resultados esperados para el cumplimiento de las metas relacionadas con la PNA. Es necesario evaluar los currículos, los PRAU y los programas ambientales disponibles en las universidades, no solo desde las exigencias normativas, sino desde los procesos psicológicos asociados con el verdadero aprendizaje, entendido como el cambio estable en el comportameintoproambiental de los estudiantes.

\section{Referencias bibliográficas}

Alvarez-Suárez, P., Vega-Marcote, P. y García Mira, R. (2013). Sustainable consumption: a teaching intervention in higher education. International Journal of Sustainability in Higher Education, 115(1),3-15.

Ajzen, I. (1991). The theory of planned behavior. Organizational Behavior and Human Decision Processes, 50, 179-211.

Ajzen, I. (2002) Perceived behavioral control, self-efficacy, locus of control, and the Theory of Planned Behavior.Journal of Applied Social Psychology, 32(4), 665-683. doi: 10.1111/ j.1559-1816.2002.tb00236.

Ajzen, I., y Fishbein, M. (2005). The influence of attitudes on behavior.TheHandbook of Attitudes, 173(221), 31.

Amérigo, M. (2006). La investigación en España sobre actitudes proambientales y comportamiento ecológico. Medio Ambiente y Comportamiento Humano, 7(2), 45-71. 
Berenguer, J. y Corraliza, J. (2000). Preocupación ambiental y comportamientos ecológicos. Psicothema, 12(3), 325-329.

Chawla, L. y Derr, V. (2012). The Development of Conservation Behaviors in Childhood and Youth. En S. Clayton (Ed.), The Oxford Handbook of Environmental and Conservation and Psychology (pp. 527-555). Oxford: Oxford UniversityPress.

Corral-Verdugo, V. (2010).Psicología de la sustentabilidad. México: Trillas

Corral-Verdugo, V. (2012a). Towards a positive psychology of sustainability.Bulletin of People-Environment Studies, 38, 4-8.

Corral-Verdugo, V. (2012b). The positive Psychology of Sustainability. Environment, Development and Sustainability, 14, 651-666.

Corral-Verdugo, V. y Pinheiro, J. (2004). Aproximaciones al estudio de la conducta sustentable. Medio Ambiente y Comportamiento Humano, 5(1-2), 1-26.

Corral-Verdugo, V. y Pinheiro, J. (2009).Environmentalpsychologywith a Latin American taste. Journal of Environmental Psychology, 29, 366-374.

Corral-Verdugo, V., Tapia, C., García, F., Varela, C., Cuen, A., y Barrón, M. (2012). Validation of a scale assessing psychological restoration associated with sustainable behaviours. Psyecology, 3, 87-100.

Cortés-Peña, O. (2011a). Actitudes proambientales y el consumo sustentable de los servicios públicos domiciliarios de agua y energía eléctrica en la ciudad de Barranquilla(Tesis de Grado. Maestría en Psicología). Universidad del Norte, Barranquilla,Colombia.

Cortés-Peña, O.F. (2011b). Comportamiento proambiental y pensamiento económico en la construcción del desarrollo sostenible. Cultura Educación y Sociedad, 2(1), 43-56.

Cortés-Peña, O. (2016a). Comportamiento proambiental y desarrollo económico sustentable en jóvenes universitarios. Opción, 32(9), 387- 407.

Cortés-Peña, O. (2016b).Sustainable development in synergistic relationship with pro-ambient behavior and fair trade.International Journal of Management Sciences and Opera- tions Research, 1(1), 54-58. doi:10.17981/ ijmsor.01.01.08

Crissien-Borrero, T., Cortés-Peña, O., y Herrera-Mendoza, K. (2016). Pro-Environmental Assessment and Sustainable Consumption of Household Public Services in Barranquilla Colombia.The European Proceedings of Social \& Behavioural Sciences, 17, 434-440. doi: 10.15405/epsbs.2016.11.02.39

Dunlap, R.E., Van Liere, K.D., Merting, A.G. y Jones, R.E. (2000). Measuringendorsement of the New EcologicalParadigm: A revised NEP scale. Journal of Social Issues, 56(3), 425-442.

Fishbein, M. y Ajzen, I. (2011).Predicting and changing behavior: The reasoned action approach. New York: Taylor \& Francis.

García-Mira, R. (2009) Sostenibilidad y cultura ambiental: aspectos psicosociales, educativos y de participación pública. En R. García-Mira y P. Vega Marcote(Eds.),Sostenibilidad, valores y cultura ambiental (pp. 23-30). Madrid: EdicionesPirámide.

Garzón-Medina, C., Barreto, I. y Sandoval-Escobar. M. (2018). Efectividad de un programa de comportamiento sustentable en la intención de conducta proambiental de alimentos transgénicos y marcas verdes. En D. Forero (Ed), Elección, Razonamiento y Decisión (pp. 237-252). Bogotá: Konrad Lorenz Editores.

Gifford, R. (2014). Environmental psychology matters. Annual Review of Psychology, 65, 541-579. doi: 10.1146/annurev-psych-010213-115048.

Gifford, R. y Sussman, R. (2012). Environmental attitudes.En S. Clayton (Ed.),Handbook of environmental and conservation psychology. Oxford, UK: Oxford UniversityPress.

González-López, A. (2002). La preocupación por la calidad del medio ambiente: un modelo cognitivo sobre la conducta ecológica [Tesis doctoral inédita]. Universidad Complutense, Madrid. Disponible en http://biblioteca.ucm. es/tesis/psi/ucm-t26479.pdf

Medina, I. y Páramo, P. (2014). La investigación en educación ambiental en América Latina: un análisis bibliométrico. Revista Colombiana de Educación, 66, 19-72. 
Páramo, P. y Gómez, F. (1997) Actitudes hacia el medio ambiente: su medición a partir de las teorías de facetas. Revista Latinoamericana de Psicología, 29(2), 243-266.

Páramo, P., Sandoval-Escobar, M., Jakovcevic, A., Ferreiro, J., Mustaca, A., Jengich, A., Brenes, J., Ortega-Andeane, P., Vivas, F., Moros, O., Méndez, C., Pasquali, C., Bassani, M., Anicama, J., Castillo, B., Urzúa, A. y Denegri, M. (2015). Assessment of environmental quality, degree of optimism, and the assignment of responsibility regarding the state of the environment in Latin America.UniversitasPsychologica, 14(2), 605-618. doi:10.11144/Javeriana.upsy14-2.aeqd

Paternina, L. (2007). Correlación entre las variables cognitivas: conciencia de las consecuencias ambientales, creencias ecológicas, negación de la obligación, norma personal, control ambiental y valores, y la conducta ecológica en habitantes de la ciudad de Barranquilla mayores de 18 anos. [Tesis de maestría]. Universidad del Norte, Colombia.

Rodríguez-González., L. y Sandoval-Escobar. M. (2018). Comparación de la elección de productos ecológicos y no ecológicos: influencia de la retroalimentación negativa y la actividad previa. En D. Forero (Ed), Elección, Razonamiento y Decisión (pp. 207-236). Bogotá: Konrad Lorenz Editores.

Román, Y. (2014). Sistema Ambiental Universitario: Modelo Integrado de Gestión para la inclusión de la Dimensión Ambiental y Urbana en la Educación Superior. Bogotá: Universidad Piloto de Colombia.

Sandoval, M. (2012) Comportamiento sustentable y educación ambiental: una visión desde las prácticas culturales. Revista Latinoamericana de Psicología, 44(1), 181-196.

Sandoval, M. y Barreto, I. (2014). Inventario de Comportamiento Proambiental (ICP). Proyecto COLCIENCIAS. Análisis longitudinal de los factores determinantes de la efectividad de un programa de comportamiento sustentable (CS) en el desarrollo de patrones de compra, uso y disposición de bienes de consumo y servicios públicos en hogares bogotanos- Convenio 492. Bogotá, Colombia: Fundación Universitaria Konrad Lorenz.

Sapiains, R.y Ugarte, A. (2017a). Contribuciones de la Psicología al abordaje de la dimensión humana del cambio climático en Chile (Primera parte). Interdisciplinaria, 34(1), 91-105.doi: 10.16888/interd.2017.34.1.6

Sapiains, R., y Ugarte, A. (2017b). Contribuciones de la psicología al abordaje de la dimensión humana del cambio climático en Chile (Segunda Parte). Interdisciplinaria, 34(2), 259-274.doi: 10.16888/interd.2017.34.2.2

Smith-Sebasto, N.J. y Fortner, R. (1994).TheEnvironmentalActionInternal Control IndexArticle. TheJournal of EnvironmentalEducation, 25(4), 23-29.

Schwartz, S.H. (1994). Beyondindividualism/ collectivism: New Cultural dimensions of Values. EnU.Kim, H.C. Triandis, C. Kagitcibasi, S.C. Choi y G. Yoon (Eds.), Individualism and Collectivism: Theory, Method and Applications (pp. 85-119). ThousandOaks, CA: Sage.

Torres-Hernández, M., Barreto, I. y Rincón, J. (2015). Creencias y normas subjetivas como predictores de intención de comportamiento proambiental.Suma Psicológica, 22(2), 86-92.

Uzzell, D. (2000b). Thepsycho-spatialdimension to global environmental problems.Journal of EnvironmentalPsychology, 20(4), 307- 318.

Uzzell, D., Pol, E. y Badenas, D. (2002). Place identification, social cohesion and environmentalsustainability.Environment and Behavior, 34(1), 26-53.

Vlek, C. y Steg, L. (2007). Human behavior and environmentalsustainability: Problems, drivingforces and researchtopics. Journal of Social Issues, 63(1), 1-19.

Recibido: 5 de diciembre de 2017 Aceptado: 26 de septiembre de 2019 
Sandoval-Escobar, Páramo, Orejuela, González Gallo, Cortés, Herrera Mendoza, Garzón y Erazo 